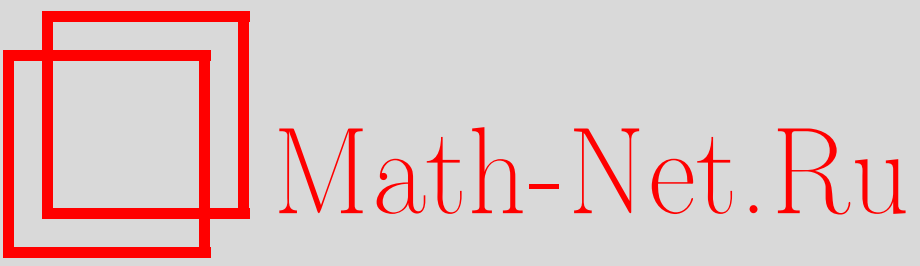

А. П. Исаев, О. В. Огиевецкий, БРСТ-оператор для квантовых алгебр Ли и дифференциальное исчисление на квантовых группах, ТМФ, 2001, том 129, номep 2, 298-316

DOI: https://doi.org/10.4213/tmf537

Использование Общероссийского математического портала Math-Net.Ru подразумевает, что вы прочитали и согласны с пользовательским соглашением

http://www . mathnet.ru/rus/agreement

Параметры загрузки:

IP: 54.197 .217 .227

26 апреля 2023 г., 17:57:01 


\author{
ТЕОРЕТИЧЕСКАЯ \\ И МАТЕМАТИЧЕСКАЯ \\ ФИЗИКА \\ Том 129, № 2 \\ ноябрь, 2001
}

(C) 2001 г.

А.П. Исаев ${ }^{*}$, О.В. Огиевецкий ${ }^{\dagger}$

\title{
БРСТ-ОПЕРАТОР ДЛЯ КВАНТОВЫХ АЛГЕБР ЛИ И ДИФФЕРЕНЦИАЛЬНОЕ ИСЧИСЛЕНИЕ НА КВАНТОВЫХ ГРУППАХ
}

Для алгебры Хопфа $\mathcal{A}$ определена структура дифференциальных комплексов на двух дуальных внешних алгебрах Хопфа: 1) внешнем расширении $\mathcal{A}$ и 2) внешнем расширении дуальной алгебры $\mathcal{A}^{*}$. Дубль Гейзенберга этих двух внешних алгебр Хопфа определяет дифференциальную алгебру для дифференциального исчисления Картана на алгебре $\mathcal{A}$. Первый дифференциальный комплекс - это аналог комплекса де Рама. В случае, когда $\mathcal{A}^{*}$ является универсальной обертывающей (супер)алгебры Ли, второй комплекс совпадает со стандартным комплексом. Дифференциал реализуется как (анти)коммутатор с БРСТ-оператором $Q$. Приведено рекуррентное соотношение, которое однозначно определяет оператор $Q$. Для случая квантовой алгебры Ли $U_{\mathrm{q}}(g l(N))$ явно построены БРСТ- и анти-БРСТ-операторы и сформулирована теорема о разложении Ходжа.

\section{1. ВВЕДЕНИЕ}

Теория биковариантного дифференциального исчисления на алгебрах Хопфа $\mathcal{A}$ была разработана Вороновичем [1] (в качестве обзоров мы рекомендуем [2]-[4]) на основе аксиоматики некоммутативной дифференциальной геометрии, предложенной Конном [5]. Затем в [6], [7] было показано, что теория Вороновича применима для описания дифференциальных исчислений на квантовых аналогах некоторых групп Ли и может быть адаптирована для $R$-матричного формализма, предложенного в работе [8]. Специальньй пример $\operatorname{Fun}\left(G L_{\mathrm{q}}(N)\right)$ детально рассматривался в [6], [9]-[11] (см. также [4] и ссылки, приведенные в этом обзоре).

В работах [4], [6], [11] было установлено, что $G L_{\mathrm{q}}(N)$-дифференциальная алгебра есть алгебра Пуанкаре-Биркгофа-Витта (ПБВ) и, следовательно, действительно описывает квантовую деформацию классической дифференциальной алгебры на $G L(N)$.

\footnotetext{
* Объединенный институт ядерных исследований, Дубна, Московская обл., Россия. E-mail: isaevap@thsun1.jinr.ru

${ }^{\dagger}$ Center of Theoretical Physics, Luminy, Marseille, France; Физический институт им. П. Н. Лебедева РАН, Москва, Россия. E-mail: oleg@cpt.univ-mrs.fr
} 
Дифференциальная алгебра ПБВ-типа на $S L_{\mathrm{q}}(N)$ была построена в работе [12] (см. также [13]).

Однако дальнейший анализ (см. [14]) показал, что биковариантные дифференциальные алгебры [7], [15] исчисления Вороновича на Fun $\left(S O_{\mathrm{q}}(N)\right)$ и Fun $\left(S p_{\mathrm{q}}(2 n)\right)$ (в действительности, их биковариантные подалгебры дифференциальных 1-форм) имеют "неправильные" размерности, т.е. не являются ПБВ-подалгебрами. Таким образом, эти некоммутативные алгебры не могут интерпретироваться как квантовые деформации соответствующих классических дифференциальных алгебр.

В данной статье мы связываем теорию Вороновича с деформацией БРСТ-теории (обзор по БРСТ-теории см. в [16]; обсуждение связи БРСТ-теории с теорией когомологий алгебр Ли можно найти в [17], [18]). Эта связь уже упоминалась в [19], [20] в контексте обсуждения так называемых "квантово-групповых калибровочных теорий" (о квантово-групповых калибровочных теориях и соответствуюшей некоммутативной геометрии см. [21], а также ссылки, приведенные там). Главная идея заключается в том, что в теории Вороновича внешний дифференциал $d$, действуюший на внешнем расширении алгебры Хопфа $\mathcal{A}$, должен генерироваться нильпотентным оператором, представляющим собой не что иное, как БРСТ-заряд, связанный с деформированной алгеброй векторных полей на $\mathcal{A}$. Построение явной формулы для этого БРСТ-оператора и есть главная цель данной статьи.

Во втором разделе мы вводим понятие квантовой алгебры Ли. В третьем разделе собраны результаты о картановском дифференциальном исчислении на алгебрах Хопфа (это исчисление есть расширение дифференциального исчисления Вороновича) и показано, как квантовые алгебры Ли естественно возникают в рамках этих исчислений. БРCТ-оператор для квантовых алгебр Ли строится в разделе 4 . В разделе 5 мы детально рассматриваем специальный случай квантовой алгебры Ли $U_{\mathrm{q}}(g l(N))$. В этом случае нам удается явно построить БРСТ- и анти-БРСТ-операторы и сформулировать теорему о разложении Ходжа.

\section{2. КВАНТОВЫЕ АЛГЕБРЫ ЛИ}

Квантовая алгебра Ли (см. [1], [2], [19], [22]) определяется двумя тензорами $-C_{i j}^{k}$ и $\sigma_{i j}^{m k}$ (индексы принадлежат некоторому множеству $\mathcal{N}$, скажем $\mathcal{N}=\{1, \ldots, N\}$ ). По определению матрица $\sigma_{i j}^{m k}$ имеет собственное значение 1 ; требуется, чтобы $\left(P_{(1)}\right)_{i j}^{m k} \times$ $C_{m k}^{n}=0$, где $P_{(1)}-$ проектор на подпространство собственных векторов оператора $\sigma$, отвечающих собственному значению 1.

По определению квантовая алгебра Ли порождается элементами $\chi_{i}, i=1, \ldots, N$, которые удовлетворяют соотношениям

$$
\chi_{i} \chi_{j}-\sigma_{i j}^{m k} \chi_{m} \chi_{k}=C_{i j}^{k} \chi_{k} .
$$

Здесь структурные константы $C_{i j}^{k}$ подчиняются условиям 


$$
\begin{gathered}
C_{n i}^{p} C_{p j}^{l}=\sigma_{i j}^{m k} C_{n m}^{p} C_{p k}^{l}+C_{i j}^{p} C_{n p}^{l} \Leftrightarrow \\
\Leftrightarrow C_{|12\rangle}^{\langle 1|} C_{|13\rangle}^{\langle 4|}=\sigma_{23} C_{|12\rangle}^{\langle 1|} C_{|13\rangle}^{\langle 4|}+C_{|23\rangle}^{\langle 3|} C_{|13\rangle}^{\langle 4|}, \\
C_{n i}^{k} \sigma_{k q}^{p m}=\sigma_{i q}^{s j} \sigma_{n s}^{p k} C_{k j}^{m} \Leftrightarrow C_{|12\rangle}^{\langle 1|} \sigma_{13}=\sigma_{23} \sigma_{12} C_{|23\rangle}^{\langle 3|}, \\
\left(\sigma_{i m}^{p j} C_{q p}^{n}+\delta_{\mathrm{q}}^{n} C_{i m}^{j}\right) \sigma_{n j}^{k s}=\sigma_{q i}^{j n}\left(\sigma_{n m}^{p s} C_{j p}^{k}+\delta_{j}^{k} C_{n m}^{s}\right) \Leftrightarrow \\
\Leftrightarrow\left(\sigma_{23} C_{|12\rangle}^{\langle 1|}+C_{|23\rangle}^{\langle 3|}\right) \sigma_{13}=\sigma_{12}\left(\sigma_{23} C_{|12\rangle}^{\langle 1|}+C_{|23\rangle}^{\langle 3|}\right) .
\end{gathered}
$$

Матрица $\sigma_{i j}^{m k}$ удовлетворяет уравнению Янга-Бакстера

$$
\sigma_{i_{1} i_{2}}^{j_{1} j_{2}} \sigma_{j_{2} i_{3}}^{n_{2} k_{3}} \sigma_{j_{1} n_{2}}^{k_{1} k_{2}}=\sigma_{i_{2} i_{3}}^{j_{2} j_{3}} \sigma_{i_{1} j_{2}}^{k_{1} n_{2}} \sigma_{n_{2} j_{3}}^{k_{2} k_{3}} \Leftrightarrow \sigma_{12} \sigma_{23} \sigma_{12}=\sigma_{23} \sigma_{12} \sigma_{23}
$$

В правых частях выражений (2)-(5) мы использовали матричные обозначения Фаддеева-Решетихина-Тахтаджяна [8]; индексы $\{1,2,3, \ldots\}$ - номера векторных пространств, например $f_{1}=f_{j_{1}}^{i_{1}}$ обозначает матрицу, которая действует в первом векторном пространстве. В добавление к этому мы используем "входящие" и "выходящие" индексы, например $\Omega^{\langle 1|}=\Omega^{i_{1}}$ и $\gamma_{|1\rangle}=\gamma_{j_{1}}$ обозначают ковектор с одним "выходяшим" индексом и вектор с одним "входящим" индексом, соответственно. Таким образом, в этих обозначениях матрица $f_{1}$ может быть записана в виде $f_{1}=f_{|1\rangle}^{\langle 1|}$.

Заметим, что соотношения (2)-(5) могут быть оправданны, если мы рассмотрим моном $\chi_{|1\rangle} \chi_{|2\rangle} \chi_{|3\rangle}$ третьей степени и переупорядочим его двумя разными способами, используя определяюшие соотношения (1). Требование совпадения результатов этих двух переупорядочений дает соотношение

$$
\begin{aligned}
0= & \left(\sigma_{12} \sigma_{23} \sigma_{12}-\sigma_{23} \sigma_{12} \sigma_{23}\right) \chi_{|1\rangle} \chi_{|2\rangle} \chi_{|3\rangle}+ \\
& +\left[\left(\sigma_{23} C_{|12\rangle}^{\langle 1|}+C_{|23\rangle}^{\langle 3|}\right) \sigma_{13}-\sigma_{12}\left(\sigma_{23} C_{|12\rangle}^{\langle 1|}+C_{|23\rangle}^{\langle 3|}\right)\right] \chi_{|1\rangle} \chi_{|3\rangle}- \\
& -\left[C_{|12\rangle}^{\langle 1|} \sigma_{13}-\sigma_{23} \sigma_{12} C_{|23\rangle}^{\langle 3|}\right] \chi_{|1\rangle} \chi_{|3\rangle}- \\
& -\left(\left(1-\sigma_{23}\right) C_{|12\rangle}^{\langle 1|}+C_{|23\rangle}^{\langle 3|}\right) C_{|13\rangle}^{\langle 4|} \chi_{|4\rangle} .
\end{aligned}
$$

Это действительно тождество: кубический член исчезает в силу (5), квадратичные члены обрашаются в нуль вследствие (4) и (3), а последний член исчезает благодаря тождеству Якоби (2).

ЗАМЕчАНИЕ 1. Заметим, что для получения тождества (6) не обязательно в точности требовать выполнения соотношений (3) и (4), т.к. только их комбинация входит в (6). Для того чтобы получить соотношения (3) и (4) как условия совместности, необходимо рассмотреть следуюшую ситуацию. Для каждого целого $M$ пусть $\chi_{i}^{(M)}$ будет копией генераторов $\chi_{i}$. Соотношения между различными копиями зададим в виде

$$
\chi_{|1\rangle}^{(K)} \chi_{|2\rangle}^{(M)}=\sigma_{12} \chi_{|1\rangle}^{(M)} \chi_{|2\rangle}^{(K)}+C_{|12\rangle}^{\langle 3|} \chi_{|3\rangle}^{(K)} \text { для } K<M .
$$


Тогда, упорядочивая двумя различными способами выражение $\chi_{|1\rangle}^{(L)} \chi_{|2\rangle}^{(M)} \chi_{|3\rangle}^{(K)}$ с $L<$ $M<K$, получим уравнение (5) при кубических членах по $\chi$, а при членах более низкого порядка возникает тождество

$$
\begin{aligned}
{\left[\left(\sigma_{23} C_{|12\rangle}^{\langle 1|}+C_{|23\rangle}^{\langle 3|}\right) \sigma_{13}-\sigma_{12}\left(\sigma_{23} C_{|12\rangle}^{\langle 1|}+C_{|23\rangle}^{\langle 3|}\right)\right] \chi_{|1\rangle}^{(M)} \chi_{|3\rangle}^{(L)}-} \\
-\left[C_{|12\rangle}^{\langle 1|} \sigma_{13}-\sigma_{23} \sigma_{12} C_{|23\rangle}^{\langle 3|}\right] \chi_{|1\rangle}^{(K)} \chi_{|3\rangle}^{(L)}- \\
-\left(\left(1-\sigma_{23}\right) C_{|12\rangle}^{\langle 1|}-C_{|23\rangle}^{\langle 3|}\right) C_{|13\rangle}^{\langle 4|} \chi_{|4\rangle}^{(L)}=0 .
\end{aligned}
$$

Это тождество полностью эквивалентно набору соотношений (2)-(4).

ЗАмечАниЕ 2. Тождество Якоби (2) обеспечивает существование присоединенного представления, в котором генератор $\chi_{i}$ реализуется матрицей $\left(\operatorname{ad}\left(\chi_{i}\right)\right)_{k}^{j}=C_{k i}^{j}$. Кроме того, каждая квантовая алгебра Ли обладает тривиальным одномерным представлением, в котором каждый генератор $\chi_{i}$ действует как ноль.

ЗАмЕчАнИЕ 3. Квантовые алгебры Ли с определяющими соотношениями (1)-(5) обобщают обычные (супер)алгебры Ли. В недеформированном случае, когда $\sigma_{i j}^{m k}=$ $(-1)^{(m)(k)} \delta_{j}^{m} \delta_{i}^{k}-$ матрица суперперестановки (здесь $(m)=0,1(\bmod 2)$ обозначает четность генератора $\chi_{m}$; в этом случае (5) выполняется автоматически и $\sigma^{2}=1$ ), уравнения (1) и (2) соответственно совпадают с определяющими соотношениями и тождествами Якоби для (супер)алгебр Ли. Наконец, уравнение (3) эквивалентно условию

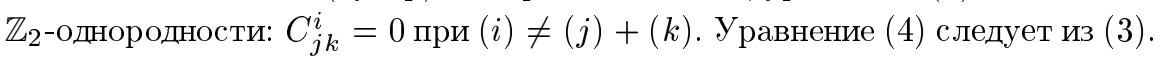

ЗАмЕчаниЕ 4. В [19] было замечено, что соотношения (2)-(5) могут быть записаны как уравнения Янга-Бакстера для матришы $S_{C D}^{A B}$, где заглавные латинские индексы $A, B, \ldots$ принадлежат набору $0 \cup \mathcal{N}=\{0,1, \ldots, N\}$ (у матрицы $\sigma_{k l}^{i j}$ индексы принадлежат набору $\mathcal{N})$. Матрица $S$ определяется соотношениями

$$
S_{k l}^{i j}=\sigma_{k l}^{i j}, \quad S_{k l}^{0 j}=C_{k l}^{j}, \quad S_{B 0}^{0 A}=\delta_{B}^{A}, \quad S_{0 B}^{A 0}=\delta_{B}^{A},
$$

а остальные компоненты $S$ - нули.

\section{3. ДИФФЕРЕНЦИАЛЬНОЕ ИСЧИСЛЕНИЕ КАРТАНА НА АЛГЕБРАХ ХОПФА}

В этом разделе мы показываем, что квантовые алгебры Ли (1) естественно возникают (как квантовые аналоги векторных полей) в контексте биковариантного дифференциального исчисления на алгебрах Хопфа.

3.1. Внешние алгебры Хопфа. Пусть $\mathcal{A}(\Delta, \epsilon, S)$ - алгебра Хопфа и $\mathcal{A}^{*}$ - дуальная к $\mathcal{A}$ алгебра Хопфа. Коумножение, а также левое и правое $\mathcal{A}$-кодействия на $\mathcal{A}$ имеют вид

$$
\Delta(a)=a_{(1)} \otimes a_{(2)} \equiv \Delta_{\mathrm{L}, \mathrm{R}}(a), \quad a \in \mathcal{A} .
$$


Здесь для коумножения использована стандартная запись Свидлера. Над алгеброй $\mathcal{A}$ можно определить (см. [1]) биковариантный бимодуль (бимодуль Хопфа) $\Gamma^{(1)}$ как линейное пространство с левоинвариантными базисными элементами $\left\{\omega^{i}\right\}$ такими, что левое и правое $\mathcal{A}$-кодействия на $\Gamma^{(1)}$ имеют вид

$$
\Delta_{\mathrm{L}}\left(\omega^{i}\right)=1 \otimes \omega^{i}, \quad \Delta_{\mathrm{R}}\left(\omega^{i}\right)=\omega^{j} \otimes r_{j}^{i}
$$

где $r_{j}^{i} \in \mathcal{A}$. Так как любой левоинвариантный элемент в $\Gamma^{(1)}$ может быть записан как линейная комбинация базисных элементов $\left\{\omega^{i}\right\}$, то справедливо разложение $S\left(a_{(1)}\right) \times$ $\omega^{i} a_{(2)}=f_{j}^{i}(a) \omega^{j}$, которое переписывается в виде коммутационного соотношения элементов $a \in \mathcal{A}$ с элементами $\omega^{i} \in \Gamma^{(1)}$ :

$$
\omega^{i} a=\left(f_{j}^{i} \triangleright a\right) \omega^{j}=a_{(1)} f_{j}^{i}\left(a_{(2)}\right) \omega^{j}
$$

где $f_{j}^{i} \in \mathcal{A}^{*}$. Ковариантность (12) по отношению к правому $\mathcal{A}$-кодействию $(10),(11)$ приводит к основному соотношению на элементы $f$ и $r$ :

$$
\left(f_{i}^{j} \triangleright a\right) r_{k}^{i}=r_{i}^{j}\left(a \triangleleft f_{k}^{i}\right) \quad \forall a \in \mathcal{A}
$$

Как было показано в [23] (более детальное рассмотрение проведено в [3]), можно расширить алгебру Хопфа $\mathcal{A}$ до внешней алгебры Хопфа $\Gamma^{\wedge}=\left\{\mathcal{A} \oplus \Gamma^{(1)} \oplus \Gamma^{(2)} \oplus \cdots\right\}$ с помошью определения Вороновича для ковариантного внешнего произведения

$$
\omega^{i} \wedge \omega^{j}=\omega^{i} \otimes \omega^{j}-\omega^{k} \otimes \omega^{l} \sigma_{k l}^{i j} \Rightarrow \omega^{\langle 1|} \wedge \omega^{\langle 2|}=\omega^{\langle 1|} \otimes \omega^{\langle 2|}(1-\sigma)_{12}
$$

где матрица $\sigma_{k l}^{i j}=f_{l}^{i}\left(r_{k}^{j}\right)$ (аналог матрицы перестановки) удовлетворяет уравнению Янга-Бакстера (5), которое следует из (13), если положить $a=r_{n}^{m}$ и затем вычислить спаривание с $f_{p}^{l}$ (при этом необходимо воспользоваться явными выражениями для $\Delta\left(f_{j}^{i}\right)$ и $\Delta\left(r_{j}^{i}\right)$, см. ниже).

Обобшение внешнего произведения (14) на случай произведения $n$ 1-форм дается формулой

$$
\omega^{\langle 1|} \wedge \omega^{\langle 2|} \wedge \cdots \wedge \omega^{\langle n|}=\omega^{\langle 1|} \otimes \omega^{\langle 2|} \otimes \cdots \otimes \omega^{\langle n|} A_{1 \rightarrow n}
$$

Здесь матричный оператор $A_{1 \rightarrow n}$ - аналог антисимметризатора в $n$ пространствах. Этот оператор определяется индуктивно (см., например, [24]):

$$
A_{1 \rightarrow n}=\left(1-\sum_{k=1}^{n-1}(-1)^{n-k-1} \sigma_{k \rightarrow n}\right) A_{1 \rightarrow n-1}=\left(1-\sum_{k=2}^{n}(-1)^{k} \sigma_{k \leftarrow 1}\right) A_{2 \rightarrow n}
$$

где $\sigma_{n \leftarrow k}=\sigma_{n-1} \ldots \sigma_{k+1 k+2} \sigma_{k k+1}, \sigma_{k \rightarrow n}=\sigma_{k k+1} \sigma_{k+1 k+2} \ldots \sigma_{n-1 n}(n>k)$. Ecли последовательность операторов $A_{1 \rightarrow n}$ обрывается на шаге $n=h+1\left(A_{1 \rightarrow n}=0\right.$ для любого $n>h)$, то число $h$ называется высотой матрицы $\sigma_{12}$. 
Расширение коумножения (10) на всю внешнюю алгебру $\Gamma^{\wedge}$ определяется формулами [23]

$$
\begin{gathered}
\Delta\left(\omega^{i}\right) \equiv \Delta_{\mathrm{L}}\left(\omega^{i}\right)+\Delta_{\mathrm{R}}\left(\omega^{i}\right)=1 \otimes \omega^{i}+\omega^{j} \otimes r_{j}^{i}, \\
\Delta(\omega a)=\Delta(\omega) \Delta(a), \quad \Delta(a \omega)=\Delta(a) \Delta(\omega),
\end{gathered}
$$

где $\otimes-$ градуированное тензорное произведение, а градуировка фиксируется соотношениями: $\operatorname{deg}(\omega)=n$ для $\omega \in \Gamma^{(n)}$. Условие ассоциативности для $\omega a b$ по отношению к коммутационному соотношению (12) и условие коассоциативности для (17) приводят к формулам коумножений для элементов $f_{j}^{i}$ и $r_{j}^{i}$ :

$$
\Delta\left(f_{j}^{i}\right)=f_{k}^{i} \otimes f_{j}^{k}, \quad \Delta\left(r_{j}^{i}\right)=r_{j}^{k} \otimes r_{k}^{i}
$$

Другие структурные отображения для $f, r$ и $\omega$ получаются из (17), (19):

$$
\begin{gathered}
\epsilon\left(r_{j}^{i}\right)=\delta_{j}^{i}=\epsilon\left(f_{j}^{i}\right), \quad S\left(f_{k}^{i}\right) f_{j}^{k}=\delta_{j}^{i}=S\left(r_{j}^{k}\right) r_{k}^{i}, \\
\epsilon\left(\omega^{i}\right)=0, \quad S\left(\omega^{i}\right)=-\omega^{j} S\left(r_{j}^{i}\right) .
\end{gathered}
$$

Резюмируя этот пункт, подчеркнем, что знание двух наборов элементов $\left\{r_{j}^{i}\right\} \in \mathcal{A}$ и $\left\{f_{j}^{i}\right\} \in \mathcal{A}^{*}$, которые удовлетворяют (13), достаточно для того, чтобы построить биковариантный бимодуль над $\mathcal{A}$ и затем расширить $\mathcal{A}$ до внешней алгебры Хопфа $\Gamma^{\wedge}$.

ЗАмечАнИЕ. Пространство $\Gamma^{(k)}$ - это подпространство в $\omega^{\otimes k}$, натянутое на тензоры $a$, имеющие вид

$$
a=\omega^{i_{1}} \otimes \cdots \otimes \omega^{i_{k}} A_{i_{1} \ldots i_{k}}^{j_{1} \ldots j_{k}} a_{j_{1} \ldots j_{k}}
$$

Формула $A_{1 \ldots k} f_{1} \ldots f_{k}=f_{1} \ldots f_{k} A_{1 \ldots k}$ предполагает, что умножение элементов из $\Gamma^{k}$ и из $\mathcal{A}$ совместно с коммутационным соотношением (12). Для двух заданных форм $a \in \Gamma^{(k)}$ $($ как в $(21))$ и $b \in \Gamma^{(l)}$ (с коэффициентами $\left.b_{j_{1}} \ldots j_{l}\right)$ определим их внешнее произведение $a \wedge b \in \Gamma^{(k+l)}$ как

$$
a \wedge b=\omega^{i_{1}} \otimes \cdots \otimes \omega^{i_{k+l}} A_{i_{1} \ldots i_{k+l}}^{j_{1} \ldots j_{k+l}} c_{j_{1} \ldots j_{k+l}},
$$

где

$$
c_{i_{1} \ldots i_{k} j_{1} \ldots j_{l}}=a_{i_{1} \ldots i_{k}} b_{j_{1} \ldots j_{l}} .
$$

Определение (22), (23) внешнего произведения дифференциальных форм самосогласованно. Действительно, можно изменить $a$, добавив такое $\delta a$, что $A_{1 \ldots k} \delta a=0$. Тогда $A_{1 \ldots k+l} \delta a \wedge b=0$, т.к. $A_{1 \ldots k+l}$ (в силу (16)) пропорционально $A_{1 \ldots k}$, т.е. $A_{1 \ldots k+l}=Z$. $A_{1 \ldots k}$ для некоторого $Z$. Прямая проверка показывает, что это тензорное произведение ассоциативно. 
3.2. Дуальная внешняя алгебра Хопфа. По аналогии с конструкцией предыдущего пункта можно определить биковариантный бимодуль $\Gamma^{(1) *}$ и соответствующую внешнюю алгебру Хопфа над дуальной алгеброй $\mathcal{A}^{*}\left(\Delta(h)=h_{(1)} \otimes h_{(2)}, h \in \mathcal{A}^{*}\right)$, вводя два набора элементов $\left\{\bar{r}_{j}^{i}\right\} \in \mathcal{A}$ и $\left\{\bar{f}_{j}^{i}\right\} \in \mathcal{A}^{*}$ таких, что (ср. с (12), (17), (20))

$$
\begin{gathered}
\gamma_{i} h=\left(\bar{r}_{i}^{j} \triangleright h\right) \gamma_{j}=h_{(1)}\left\langle h_{(2)}, \bar{r}_{i}^{j}\right\rangle \gamma_{j}, \\
\Delta\left(\gamma_{i}\right)=1 \otimes \gamma_{i}+\gamma_{j} \otimes \bar{f}_{i}^{j}, \\
\epsilon\left(\gamma_{i}\right)=0, \quad S\left(\gamma_{i}\right)=-\gamma_{j} S\left(\bar{f}_{i}^{j}\right) \Rightarrow S^{-1}\left(\gamma_{i}\right)=-S^{-1}\left(\bar{f}_{i}^{j}\right) \gamma_{j},
\end{gathered}
$$

где $\langle h, a\rangle=h(a)$ - дуальное спаривание для алгебр Хопфа $\mathcal{A}, \mathcal{A}^{*}$, а элементы $\left\{\gamma_{i}\right\}$ формируют левоинвариантный базис для бимодуля $\Gamma^{(1) *}$. Как и выше, мы получаем из $\Delta\left(\gamma_{i} h\right)=\Delta\left(\gamma_{i}\right) \Delta(h)$ соотношение (ср. с (13))

$$
\left(\bar{r}_{i}^{j} \triangleright h\right) \bar{f}_{j}^{k}=\bar{f}_{i}^{j}\left(h \triangleleft \bar{r}_{j}^{k}\right) \quad \forall h \in \mathcal{A}^{*},
$$

а внешнее произведение во внешней алгебре Хопфа $\Gamma^{\wedge *}=\mathcal{A}^{*} \oplus \Gamma^{(1) *} \oplus \Gamma^{(2) *} \oplus \cdots(\mathrm{c}$ градуировкой $\operatorname{deg}(\gamma)=-n$ для $\left.\gamma \in \Gamma^{(n) *}\right)$ определяется по формулам

$$
\gamma_{i} \wedge \gamma_{j}=\gamma_{i} \otimes \gamma_{j}-\bar{\sigma}_{i j}^{k l} \gamma_{k} \otimes \gamma_{l}, \quad \bar{\sigma}_{i j}^{k l} \equiv \bar{r}_{i}^{l}\left(\bar{f}_{j}^{k}\right)
$$

Рассмотрим специальный случай, когда $\operatorname{dim}\left(\Gamma^{(1)}\right)=\operatorname{dim}\left(\Gamma^{(1) *}\right)$, и сформулируем условия, при которых внешние алгебры Хопфа $\Gamma^{\wedge}$ и $\Gamma^{\wedge *}$ дуальны друг к другу. Спаривание алгебр $\mathcal{A}$ и $\mathcal{A}^{*}$ можно расширить до невырожденного спаривания внешних алгебр $\Gamma^{\wedge}$ и $\Gamma^{\wedge *}$ :

$$
\left\langle\gamma_{i}, \omega^{j}\right\rangle=\delta_{i}^{j}, \quad\left\langle\Gamma^{(n) *}, \Gamma^{(m)}\right\rangle \sim \delta^{n m} .
$$

Это спаривание согласовано с градуировкой. Соотношения (17), (25) и (29) приводят к уравнению

$$
\left\langle h \gamma_{i}, a \omega^{j}\right\rangle=\langle h, a\rangle \delta_{i}^{j}, \quad h \in \mathcal{A}^{*}, \quad a \in \mathcal{A} .
$$

Теперь ясно, что уравнения (29) и (30) связывают наборы элементов $\{r, f\}$ с $\{\bar{r}, \bar{f}\}$. Действительно, с одной стороны, $\left\langle\gamma_{i}, \omega^{k} a\right\rangle=\left\langle\Delta\left(\gamma_{i}\right), \omega^{k} \otimes a\right\rangle=\bar{f}_{i}^{k}(a), \forall a$, а с другой стороны, выводим $\left\langle\gamma_{i}, \omega^{k} a\right\rangle=\left\langle\gamma_{i},\left(f_{j}^{k} \triangleright a\right) \omega^{j}\right\rangle=f_{i}^{k}(a)$ и, следовательно, $\bar{f}_{i}^{k}=f_{i}^{k}$. Рассматривая спаривание $\left\langle\gamma_{i} h, \omega^{k}\right\rangle$, мы получаем $\bar{r}_{i}^{k}=r_{i}^{k}$ и, таким образом, $\bar{\sigma}_{k l}^{i j}=\sigma_{k l}^{i j}$. Это ведет к определению внешнего произведения $n$ элементов $\gamma_{i}$ в виде (см. (28)):

$$
\gamma_{|1\rangle} \wedge \gamma_{|2\rangle} \wedge \cdots \wedge \gamma_{|n\rangle}=A_{1 \rightarrow n} \gamma_{|1\rangle} \otimes \gamma_{|2\rangle} \otimes \cdots \otimes \gamma_{|n\rangle},
$$

где операторы $A_{1 \rightarrow n}$ те же самые, что и в (16). 
3.3. Дифференциал $d$ и алгебра векторных полей. Предположим, что существует дифференциальное отображение $d: \Gamma^{(n)} \rightarrow \Gamma^{(n+1)}\left(\Gamma^{(0)}=\mathcal{A}\right)$, квадрат которого равен нулю и которое удовлетворяет правилу Лейбница $\left(\omega, \omega_{i} \in \Gamma^{\wedge}\right)$ :

$$
d^{2}(\omega)=0, \quad d\left(\omega_{1} \omega_{2}\right)=d\left(\omega_{1}\right) \omega_{2}+(-1)^{\operatorname{deg}\left(\omega_{1}\right)} \omega_{1} d\left(\omega_{2}\right)
$$

Как было показано в [1], левые и правые кодействия

$$
\Delta_{\mathrm{L}}(d(a))=a_{(1)} \otimes d\left(a_{(2)}\right), \quad \Delta_{\mathrm{R}}(d(a))=d\left(a_{(1)}\right) \otimes a_{(2)}
$$

совместны с определением биковариантного бимодуля $\Gamma^{(1)}$. Из (17) мы получаем "правило Лейбница" для коумножения:

$$
\Delta(d(a))=(d \otimes \mathrm{id}+\mathrm{id} \otimes d) \Delta(a) \Rightarrow \Delta d=(d \otimes \mathrm{id}+\mathrm{id} \otimes d) \Delta .
$$

Это “правило Лейбница" может быть расширено на всю внешнюю алгебру $\Gamma^{\wedge}$ (напомним, что $\otimes-$ градуированное тензорное произведение). Так как 1-форма

$$
\omega=S\left(a_{(1)}\right) d a_{(2)} \in \Gamma^{(1)} \forall a \in \mathcal{A}
$$

левоинвариантна, то она раскладывается по левоинвариантному базису $\left\{\omega^{i}\right\}$ :

$$
S\left(a_{(1)}\right) d a_{(2)}=\sum_{i} \chi_{i}(a) \omega^{i} \Rightarrow d(a)=\left(\chi_{i} \triangleright a\right) \omega^{i}
$$

где $\chi_{i}(a)$ - некоторые коэффициенты и $\chi_{i} \in \mathcal{A}^{*}$. Применение правил Лейбница (32) и (33) к уравнению (34) дает

$$
\begin{gathered}
\Delta\left(\chi_{i}\right)=\chi_{j} \otimes f_{i}^{j}+1 \otimes \chi_{i}, \\
a \triangleleft \chi_{i}=\left(\chi_{j} \triangleright a\right) r_{i}^{j} .
\end{gathered}
$$

Уравнения (34) и (35) приводят к определениям антипода и коединицы для элементов $\chi_{i}$ :

$$
\chi_{i}(I)=\epsilon\left(\chi_{i}\right)=0, \quad S\left(\chi_{i}\right)=-\chi_{j} S\left(f_{i}^{j}\right) \Rightarrow \chi_{i}=-S\left(\chi_{j}\right) f_{i}^{j} .
$$

В соответствии с [1] элементы $\chi_{i}$ интерпретируются как векторные поля над алгеброй Хопфа $\mathcal{A}$

Коммутационные соотношения для элементов $\chi_{i}$ с произвольными $h \in \mathcal{A}^{*}$

$$
\chi_{i} h=\left(r_{i}^{k} \triangleright h\right) \chi_{k}
$$

можно получить из требования невырожденности спаривания алгебр $\mathcal{A}$ и $\mathcal{A}^{*}$. Действительно, из (36) следует

$$
\left\langle\chi_{i} h, a\right\rangle=\left\langle h, a \triangleleft \chi_{i}\right\rangle=\left\langle h,\left(\chi_{k} \triangleright a\right) r_{i}^{k}\right\rangle=\left\langle\left(r_{i}^{k} \triangleright h\right) \chi_{k}, a\right\rangle,
$$


и так как элемент $a \in \mathcal{A}$ произволен, получаем коммутационные соотношения (38). Уравнения (38) для $h=\chi_{j}$ дают определяющие соотношения (1) для элементов $\chi_{i}$ :

$$
\chi_{i} \chi_{j}=\left(r_{i}^{k} \triangleright \chi_{j}\right) \chi_{k}=\sigma_{i j}^{m k} \chi_{m} \chi_{k}+C_{i j}^{k} \chi_{k}, \quad C_{i j}^{k}=\left\langle\chi_{j}, r_{i}^{k}\right\rangle .
$$

Тождества Якоби (2) для структурных констант $C_{j k}^{i}$ могут быть получены в результате спаривания уравнения (39) с $r_{n}^{l}$. В качестве другого приложения (36) отметим, что, полагая $a=r_{j}^{i}$, можно вывести соотношение

$$
C_{n i}^{k} r_{k}^{m}=r_{n}^{k} r_{i}^{j} C_{k j}^{m} \Leftrightarrow C_{|12\rangle}^{\langle 3|} r_{3}=r_{1} r_{2} C_{|12\rangle}^{\langle 3|},
$$

которое приводит к (3) при спаривании с $f_{q}^{p}$. Уравнения (40) можно рассматривать как условия инвариантности структурных констант $C_{n i}^{k}$ относительно вращений матрицами $r_{j}^{i}$.

Уравнения (27), где $\bar{r}_{j}^{i}=r_{j}^{i}$ и $\bar{f}_{j}^{i}=f_{j}^{i}$, для $h=f_{n}^{m}$ и $h=\chi_{n}$ приводят к равенствам

$$
\begin{aligned}
\sigma_{12} f_{1} f_{2} & =f_{1} f_{2} \sigma_{12}, \\
\left(\sigma_{i m}^{p j} \chi_{p}+C_{i m}^{j}\right) f_{j}^{k} & =f_{i}^{j} f_{m}^{p} C_{j p}^{k}+f_{i}^{k} \chi_{m},
\end{aligned}
$$

и спаривание (41) и (42) с $r_{q}^{s}$ воспроизводит (5) и (4).

Введем теперь набор элементов $a^{i} \in \mathcal{A}$ таких, что $\left\langle\chi_{i}, a^{j}\right\rangle=\delta_{i}^{j}$. По определению мы имеем $S\left(a_{(1)}^{j}\right) d\left(a_{(2)}^{j}\right)=\omega^{j}$ и $\epsilon\left(a^{i}\right)=0[1]$.

Уравнение Маурера-Картана отражает тот факт, что $d^{2}(a)=0$. Это уравнение может быть выведено следуюшим образом:

$$
\begin{aligned}
d \omega^{k} & =d\left(S\left(a_{(1)}^{k}\right) d\left(a_{(2)}^{k}\right)=-S\left(a_{(1)}^{k}\right) d\left(a_{(2)}^{k}\right) S\left(a_{(3)}^{k}\right) d\left(a_{(4)}^{k}\right)=\right. \\
& =-\chi_{i}\left(a_{(1)}^{k}\right) \chi_{j}\left(a_{(2)}^{k}\right) \omega^{i} \wedge \omega^{j}=-t_{i j}^{k} \omega^{i} \wedge \omega^{j}=-C_{i j}^{k} \omega^{i} \otimes \omega^{j},
\end{aligned}
$$

где $t_{m n}^{i} \equiv\left\langle\chi_{m} \chi_{n}, a^{i}\right\rangle$ и мы воспользовались соотношениями

$$
C_{j k}^{i}=(1-\sigma)_{j k}^{m n} t_{m n}^{i},
$$

которые могут быть получены спариванием (39) с $a^{k}$.

ЗАмЕчАнИЕ 1 . Применяя $\Delta_{\mathrm{R}}$ к первому соотношению из (34), получаем

$$
\chi_{i}\left(a_{(2)}\right) \omega^{i} \otimes S\left(a_{(1)}\right) a_{(3)}=\chi_{i}(a) \omega^{j} \otimes r_{j}^{i},
$$

в результате элементы $r_{j}^{i}$ выражаются через генераторы $a^{i}$ :

$$
\chi_{i}(a) r_{j}^{i}=S\left(a_{(1)}\right) \chi_{j}\left(a_{(2)}\right) a_{(3)} \Rightarrow r_{j}^{i}=S\left(a_{(1)}^{i}\right) \chi_{j}\left(a_{(2)}^{i}\right) a_{(3)}^{i} .
$$

С другой стороны, $\chi_{i}\left(a^{j} a\right)=f_{i}^{j}(a) \forall a \in \mathcal{A}$ и, следовательно, элементы $r_{j}^{i}$ и $f_{j}^{i}$ (которые полностью определяют $\mathcal{A}$-биковариантньй бимодуль) фиксируются элементами $a^{i} \in \mathcal{A}$ и $\chi_{i} \in \mathcal{A}^{*}$. 
ЗАмЕчАниЕ 2. Для дальнейшего рассмотрения удобно ввести слегка измененный базис векторных полей $\tilde{\chi}_{i}$ с помощью формулы $d(a)=\omega^{i}\left(\widetilde{\chi}_{i} \triangleright a\right)[25]$. Из сравнения этой формулы с (34) следуют соотношения $\chi_{i}=f_{i}^{j} \widetilde{\chi}_{j} \Rightarrow \widetilde{\chi}_{j}=S^{-1}\left(f_{j}^{i}\right) \chi_{i}$, и из (35), (37) мы получаем

$$
\tilde{\chi}_{i}=-S^{-1}\left(\chi_{i}\right) \Rightarrow \Delta\left(\widetilde{\chi}_{i}\right)=\tilde{\chi}_{i} \otimes 1+S^{-1}\left(f_{i}^{j}\right) \otimes \tilde{\chi}_{j} .
$$

Применяя $S^{-1}$ к (38), выводим

$$
h \widetilde{\chi}_{i}=\tilde{\chi}_{j}\left\langle h_{(1)}, S\left(r_{i}^{j}\right)\right\rangle h_{(2)} .
$$

3.4. Дубль Гейзенберга $\Gamma^{\wedge} \rtimes \Gamma^{\wedge *}$ и исчисление Картана. Дифференциальная алгебра для исчисления Картана должна строиться [4], [25], [26] как дубль Гейзенберга $\Gamma^{\wedge} \rtimes \Gamma^{\wedge *}$ алгебр $\Gamma^{\wedge}$ и $\Gamma^{\wedge *}$. Действие $d$ на элементы дубля Гейзенберга $\Gamma^{\wedge} \rtimes \Gamma^{\wedge *}$ также должно быть продолжено.

Прежде всего мы напомним, что дубль Гейзенберга $\Gamma^{\wedge} \rtimes \Gamma^{\wedge *}$ есть ассоциативная алгебра, которая является произведением двух дуальных алгебр $\Gamma^{\wedge}, \Gamma^{\wedge *}$ с нетривиальным $\mathbb{Z}_{2}$-градуированным правилом кросс-произведения

$$
\gamma \omega=\left(\gamma_{(1)} \triangleright \omega\right) \gamma_{(2)}=(-1)^{\operatorname{deg}\left(\gamma_{(1)}\right) \cdot \operatorname{deg}\left(\omega_{(2)}\right)} \omega_{(1)}\left\langle\gamma_{(1)}, \omega_{(2)}\right\rangle \gamma_{(2)},
$$

где $\omega \in \Gamma^{\wedge}$ и $\gamma \in \Gamma^{\wedge *}$. Это правило определяет коммутационные соотношения между элементами из $\Gamma^{\wedge}$ и элементами из $\Gamma^{\wedge *}$.

Несмотря на то что $\Gamma^{\wedge}$ и $\Gamma^{\wedge *}$ - алгебры Хопфа, их дубль Гейзенберга $\Gamma^{\wedge} \rtimes \Gamma^{\wedge *}$ не является алгеброй Хопфа. Однако $\Gamma^{\wedge} \rtimes \Gamma^{\wedge *}$ все еше обладает некоторыми свойствами ковариантного комодуля. Определим правое $\mathcal{A}$-кодействие и левое $\mathcal{A}^{*}$-кодействие на алгебру $\Gamma^{\wedge} \rtimes \Gamma^{\wedge *}$, которые сохраняют алгебраическую структуру $\Gamma^{\wedge} \rtimes \Gamma^{\wedge *}$. Обозначим через $\left\{e^{\alpha}\right\}$ и $\left\{e_{\alpha}\right\}$ базисные элементы в $\mathcal{A}^{*}$ и $\mathcal{A}$, соответственно. Правое $\mathcal{A}$-кодействие и левое $\mathcal{A}^{*}$-кодействие на $z \in \Gamma^{\wedge} \rtimes \Gamma^{\wedge *}$ определяются следуюшим образом:

$$
\Delta_{\mathrm{R}}(z)=C(z \otimes 1) C^{-1}, \quad \Delta_{\mathrm{L}}(z)=C^{-1}(1 \otimes z) C, \quad C \equiv e^{\alpha} \otimes e_{\alpha} .
$$

Аксиомы

$$
\begin{gathered}
(\mathrm{id} \otimes \Delta) \Delta_{\mathrm{R}}=\left(\Delta_{\mathrm{R}} \otimes \mathrm{id}\right) \Delta_{\mathrm{R}}, \quad\left(\mathrm{id} \otimes \Delta_{\mathrm{L}}\right) \Delta_{\mathrm{L}}=(\Delta \otimes \mathrm{id}) \Delta_{\mathrm{L}} \\
\left(\mathrm{id} \otimes \Delta_{\mathrm{R}}\right) \Delta_{\mathrm{L}}(z)=C_{13}^{-1}\left(\Delta_{\mathrm{L}} \otimes \mathrm{id}\right) \Delta_{\mathrm{R}}(z) C_{13}
\end{gathered}
$$

могут быть проверены непосредственно с использованием пентагонального тождества [27] для $C$

$$
C_{12} C_{13} C_{23}=C_{23} C_{12}
$$

Ясно, что кодействия (47) - ковариантные преобразования (гомоморфизмы) алгебры $\Gamma^{\wedge} \rtimes \Gamma^{\wedge *}$. Элемент, обратный к каноническому элементу $C$, дается формулой

$$
C^{-1}=S\left(e^{\alpha}\right) \otimes e_{\alpha}=e^{\alpha} \otimes S\left(e_{\alpha}\right),
$$


и кодействие $\Delta_{\mathrm{R}}(47)$ переписывается в виде

$$
\Delta_{\mathrm{R}}(z)=\left(e_{(1)}^{\gamma} z S\left(e_{(2)}^{\gamma}\right)\right) \otimes e_{\gamma}
$$

В частности, для $y \in \mathcal{A}^{*}$ имеем

$$
\Delta_{\mathrm{R}}(y)=y_{(1)} \otimes y_{(2)}^{\prime}=\left(e_{(1)}^{\gamma} y S\left(e_{(2)}^{\gamma}\right)\right) \otimes e_{\gamma} .
$$

Спаривая второй фактор из (49) с произвольным $x \in \mathcal{A}^{*}$, выводим соотношение

$$
y_{(1)}\left\langle x, y_{(2)}^{\prime}\right\rangle=x_{(1)} y S\left(x_{(2)}\right),
$$

которое эквивалентно коммутационным соотношениям для $x, y \in \mathcal{A}^{*}$ :

$$
x y=y_{(1)}\left\langle x_{(1)}, y_{(2)}^{\prime}\right\rangle x_{(2)},
$$

где $\Delta(x)=x_{(1)} \otimes x_{(2)}$. Обратное утверждение (что можно получить (49) из (50)) также справедливо. Сравнивая коммутационные соотношения (50) с уравнениями (45), можно найти в качестве примера правое кодействие $\Delta_{\mathrm{R}}$ на $\tilde{\chi}_{i}(49)$ :

$$
\Delta_{\mathrm{R}}\left(\tilde{\chi}_{i}\right)=\tilde{\chi}_{j} \otimes S\left(r_{i}^{j}\right), \quad \Delta_{\mathrm{R}}\left(\tilde{\gamma}_{i}\right)=\tilde{\gamma}_{j} \otimes S\left(r_{i}^{j}\right)
$$

В заключение этого раздела мы приведем кросс-коммутационные соотношения (см. (46)), которые понадобятся нам ниже:

$$
\left[\chi_{i}, \omega^{j}\right]_{-}=C_{l k}^{j} \omega^{l} f_{i}^{k}, \quad\left[\gamma_{i}, \omega^{j}\right]_{+}=f_{i}^{j}, \quad\left[\gamma_{i}, a\right]=0 \quad \forall a \in \mathcal{A} .
$$

\section{4. БРСТ-ОПЕРАТОР ДЛЯ КВАНТОВЫХ АЛГЕБР ЛИ}

В этом разделе мы найдем биинвариантный элемент $Q \in \Gamma^{\wedge} \rtimes \Gamma^{\wedge *}$ (БРСТ-оператор), который генерирует дифференциал $d$ :

$$
d \omega=[Q, \omega]_{ \pm} \forall \omega \in \Gamma^{\wedge}
$$

Оператор $Q$ должен иметь градуировку 1 и удовлетворять условию $Q \wedge Q=0$.

Прежде всего мы изменим базис дифференциальных форм $\left\{\omega^{i}\right\}$ и рассмотрим новые базисные элементы $\Omega^{i}=\omega^{j} S^{-1}\left(f_{j}^{i}\right)$, которые удобны в силу соотношений

$$
\left[\Omega^{i}, a\right]=0 \quad \forall a \in \mathcal{A} .
$$

Для $\Omega^{i}$ также справедливы (см. (52)) равенства

$$
\chi_{|2\rangle} \Omega^{\langle 2|}=\Omega^{\langle 1|}\left(\sigma_{12} \chi_{|1\rangle}+C_{|12\rangle}^{\langle 2|}\right), \quad \gamma_{|2\rangle} \Omega^{\langle 2|}=-\Omega^{\langle 1|} \sigma_{12}^{-1} \gamma_{|1\rangle}+I_{2}
$$

а определение внешнего произведения для $r$ переменных $\Omega^{i}$ дается формулой

$$
\Omega^{\langle r|} \ldots \Omega^{\langle 1|}=\Omega^{\langle r|} \otimes \cdots \otimes \Omega^{\langle 1|} A_{1 \rightarrow r}
$$

Теперь мы сформулируем главный результат данной статьи (см. также [28]). 
ПРЕДЛОЖЕНИЕ. БРСТ-оператор $Q$ для квантовой алгебры (1), который генерирует дифференциал (53), имеет следующий вид:

$$
Q=\Omega^{i} \chi_{i}+\sum_{r=1}^{h-1} Q_{(r)},
$$

где $h$ - высота оператора $\sigma_{12}\left(\right.$ см. (16)). Здесъ операторы $Q_{(r)}$ определяются как

$$
Q_{(r)}=\Omega^{\langle r+1|} \Omega^{\langle r|} \ldots \Omega^{\langle 1|} X_{|1 \ldots r+1\rangle}^{\langle\tilde{1} \ldots \tilde{r}|} \gamma_{|\tilde{1}\rangle} \ldots \gamma_{|\tilde{r}\rangle}
$$

(подразумевается внешнее произведение); $X_{|1 \ldots r+1\rangle}^{\langle 1 \ldots r|}-$ тензоры, которые удовлетворяют следующим рекуррентным соотношениям:

$$
A_{1 \rightarrow r+1} X_{|1 \ldots r+1\rangle}^{\langle 1 \ldots r|} A_{1 \rightarrow r}=A_{1 \rightarrow r+1}\left((-1)^{r} \sigma_{r+1 \leftarrow 1}-\mathbf{1}\right) X_{|2 \ldots r+1\rangle}^{\langle 2 \ldots r|} A_{2 \rightarrow r},
$$

с начальным условием $A_{12} X_{|12\rangle}^{\langle 0|}=-C_{|12\rangle}^{\langle 0|}$.

ДокАЗАТЕЛЬСтво. Мы должны проверить условия $[Q, a]=d a \quad(\forall a \in \mathcal{A})$ и $\left[Q, \omega^{i}\right]_{+}=d \omega^{i} \quad\left(\forall \omega^{i} \in \Gamma^{(1)}\right), \quad$ а также тождество $Q^{2}=0 . \quad$ Условие $[Q, a]=d a$ $(\forall a \in \mathcal{A})$ непосредственно следует из того, что $\Omega^{i}$ и $\gamma_{i}$ коммутируют со всеми $a \in \mathcal{A}$. Таким образом, нам необходимо доказать $\left[\Omega^{i} \chi_{i}, a\right]=d a$, что сразу следует из соотношений (34). Другие условия справедливы только при выполнении рекуррентных соотношений (57). Мы дадим полное доказательство этого утверждения в последуюших работах.

ЗАмечание 1 . Первое слагаемое $\Omega^{i} \chi_{i}=\omega^{i} \widetilde{\chi}_{i}$ в $(55)$ - биинвариантный элемент (см. (11), (51)). Можно доказать, что все другие члены $Q_{(r)}$ также биинвариантны.

ЗАмЕчАниЕ 2. Для произвольных $\sigma_{k l}^{i j}$ и $C_{j k}^{i}$ довольно трудно разрешить явно уравнения (57). Однако в случае $\sigma^{2}=1$ главные уравнения (57) становятся проще и полное решение для $Q$ может быть найдено. Действительно, соотношение (57) для $r=2$ дает

$$
A_{1 \rightarrow 3} X_{|123\rangle}^{\langle 12|}\left(\mathbf{1}-\sigma_{12}\right)=A_{1 \rightarrow 3}\left(\sigma_{23} \sigma_{12}-\mathbf{1}\right) X_{|23\rangle}^{\langle 2|} .
$$

Для $\sigma^{2}=1$ мы имеем $A_{1 \rightarrow 3}\left(\sigma_{23} \sigma_{12}-\mathbf{1}\right)=0$ и, следовательно, $Q_{(r)}=0$ при $r \geqslant 2$. Следовательно, БРСТ-оператор (55) имеет знакомую форму

$$
Q=\Omega^{\langle 1|} \chi_{|1\rangle}-\Omega^{\langle 2|} \otimes \Omega^{\langle 1|} C_{|12\rangle}^{\langle 1|} \gamma_{|1\rangle}
$$

В обшем случае при $\sigma^{2} \neq 1$ число слагаемых в сумме (55) ограничивается только высотой $h$ оператора $\sigma$.

Ниже мы получим явный вид оператора $Q$ для случая стандартной квантовой деформации $\mathcal{A}^{*}=U_{\mathrm{q}}(g l(N))$ универсальной обертывающей алгебры Ли $g l(N)$ (в этом случае $\left.\sigma^{2} \neq 1\right)$. 
ЗАмЕчАниЕ 3. Здесь мы приведем явные выражения для первых двух коэффициентов $X_{|123\rangle}^{\langle 12|}$ и $X_{|1234\rangle}^{\langle 123|}$. Подстановка начального условия $A_{12} X_{|12\rangle}^{\langle 0|}=-C_{|12\rangle}^{\langle 0|}$ в (58) дает

$$
A_{123} X_{|123\rangle}^{\langle 12|} A_{12}=\left[C_{2}+\sigma_{1} \sigma_{2} C_{1} \delta_{3}\right] A_{12},
$$

где мы использовали сжатые обозначения

$$
\sigma_{n}=\sigma_{n n+1}, \quad C_{n}=C_{|n n+1\rangle}^{\langle n|}, \quad \delta_{n}=\delta_{|n\rangle}^{\langle n-1|}
$$

Аналогичная формула для коэффициента $X_{|1234\rangle}^{\langle 123|}$ имеет вид

$$
A_{1234} X_{|1234\rangle}^{\langle 123|} A_{123}=\left[\left(C_{3}+\sigma_{2} \sigma_{3} C_{2} \delta_{4}\right)-\sigma_{1} \sigma_{2} \sigma_{3}\left(C_{2}+\sigma_{1} \sigma_{2} C_{1} \delta_{3}\right) \delta_{4}\right] A_{123}
$$

Для вывода (59) и (61) удобно переписать уравнения (2)-(5) в виде (см. обозначения (60)):

$$
\begin{gathered}
C_{1} \delta_{3} C_{1}=\sigma_{2} C_{1} \delta_{3} C_{1}+C_{2} C_{1}, \quad C_{1} \delta_{3} \sigma_{1}=\sigma_{2} \sigma_{1} C_{2} \\
\left(\sigma_{2} C_{1} \delta_{3}+C_{2}\right) \sigma_{1}=\sigma_{1}\left(\sigma_{2} C_{1} \delta_{3}+C_{2}\right), \quad \sigma_{1} \sigma_{2} \sigma_{1}=\sigma_{2} \sigma_{1} \sigma_{2}
\end{gathered}
$$

\section{5. БРСТ- И АНТИ-БРСТ-ОПЕРАТОРЫ ДЛЯ КВАНТОВОЙ ЛИНЕЙНОЙ АЛГЕБРЫ $U_{\mathrm{q}}(g l(N))$}

5.1. БРСТ-оператор для $U_{\mathrm{q}}(g l(N))$. Квантовая алгебра $U_{\mathrm{q}}(g l(N))$ определяется (как алгебра Хопфа) соотношениями [8]

$$
\begin{gathered}
\widehat{R} L_{2}^{ \pm} L_{1}^{ \pm}=L_{2}^{ \pm} L_{1}^{ \pm} \widehat{R}, \quad \widehat{R} L_{2}^{+} L_{1}^{-}=L_{2}^{-} L_{1}^{+} \widehat{R}, \\
\Delta\left(L^{ \pm}\right)=L^{ \pm} \otimes L^{ \pm}, \quad \varepsilon\left(L^{ \pm}\right)=\mathbf{1}, \quad S\left(L^{ \pm}\right)=\left(L^{ \pm}\right)^{-1},
\end{gathered}
$$

где элементы $(N \times N)$-матриц $\left(L^{ \pm}\right)_{j}^{i}$ - генераторы алгебры $U_{\mathrm{q}}(g l(N))$; матрицы $L^{+}$ и $L^{-}$соответственно верхне- и нижнетреугольные, а их диагональные элементы связаны соотношениями $\left(L^{+}\right)_{i}^{i}\left(L^{-}\right)_{i}^{i}=1$ для всех $i$. Матрица $\widehat{R}$ определяется как $\widehat{R}=\widehat{R}_{12}=$ $P_{12} R_{12}$ ( $P_{12}$ - матрица перестановки), а матрица $R_{12}$ обозначает стандартную $R$-матрицу Дринфельда-Джимбо для $G L_{\mathrm{q}}(N)$ :

$$
R_{12}=R_{j_{1}, j_{2}}^{i_{1}, i_{2}}=\delta_{j_{1}}^{i_{1}} \delta_{j_{2}}^{i_{2}}\left(1+(q-1) \delta^{i_{1} i_{2}}\right)+\left(q-q^{-1}\right) \delta_{j_{2}}^{i_{1}} \delta_{j_{1}}^{i_{2}} \Theta_{i_{1} i_{2}}
$$

где

$$
\Theta_{i j}=\left\{\begin{array}{l}
1 \text { при } i>j, \\
0 \text { при } i \leqslant j .
\end{array}\right.
$$

Эта $R$-матрица удовлетворяет условию Гекке $\widehat{R}^{2}=\lambda \widehat{R}+\mathbf{1}$, где $\lambda=\left(q-q^{-1}\right)$ и $q-$ параметр деформации. 
Образующие алгебры $\mathcal{A}^{*}$ определяются по формулам [4], [7], [25]

$$
\chi_{k}^{l}=\frac{1}{\lambda}\left[\left(D^{-1}\right)_{k}^{l}-\left(D^{-1}\right)_{i}^{j} f_{k j}^{l i}\right]
$$

Здесь $f_{k j}^{l i}=L^{-}{ }_{k}^{i} S\left(L^{+l}{ }_{j}\right)$, а числовая матрица $D$ может быть найдена с помощью соотношений

$$
\operatorname{Tr}_{2} \widehat{R}_{12} \Psi_{23}=P_{13}=\operatorname{Tr}_{2} \Psi_{12} \widehat{R}_{23}, \quad D_{1}=\operatorname{Tr}_{2} \Psi_{12} \Rightarrow \operatorname{Tr}_{1}\left(D_{1}^{-1} \widehat{R}^{-1}\right)=\mathbf{1}_{2},
$$

где $\operatorname{Tr}_{1}$ и $\operatorname{Tr}_{2}$ обозначают следы по первому и второму пространствам.

В рассматриваемом случае $R$-матрицы $(64)$ для $G L_{\mathrm{q}}(N)$ явное выражение для матрицы $D$ имеет вид

$$
\left(D^{-1}\right)_{j}^{i}=q^{2(N-i)+1} \delta_{j}^{i}, \quad \operatorname{Tr}\left(D^{-1} \mathbf{1}\right)=\frac{q^{2 N}-1}{q-q^{-1}} .
$$

Удобно записать коммутационные соотношения для $G L_{\mathrm{q}}(N)$-дифференциальной алгебры в терминах генераторов

$$
\begin{aligned}
L_{j}^{i} & =\left(L^{+}\right)_{k}^{i} S\left(\left(L^{-}\right)_{j}^{k}\right)=\delta_{j}^{i}-\lambda S^{-1}\left(\chi_{k}^{i}\right) D_{j}^{k}, \\
J_{n}^{i} & =-S^{-1}\left(f_{j l}^{i k}\right) \gamma_{k}^{l} D_{n}^{j}, \quad \omega_{j}^{i}=\Omega_{m}^{k} f_{k j}^{m i} .
\end{aligned}
$$

В данном случае индексы, которые использовались в предыдущих разделах, становятся парами индексов; роль элементов $\chi_{i}, \gamma_{j}$ и $\Omega^{k}$ играют образующие $\chi_{j}^{i}, \gamma_{j}^{i}$ и $\Omega_{j}^{i}$, соответственно.

Коммутационные соотношения (см. [4], [10], [25]) имеют вид

$$
\begin{aligned}
\omega_{2} \widehat{R}^{-1} \omega_{2} \widehat{R} & =-\widehat{R}^{-1} \omega_{2} \widehat{R}^{-1} \omega_{2}, & \omega_{2} \widehat{R} L_{2} \widehat{R} & =\widehat{R} L_{2} \widehat{R} \omega_{2}, \\
\omega_{2} \widehat{R} J_{2} \widehat{R}+\widehat{R} J_{2} \widehat{R} \omega_{2} & =-\widehat{R}, & L_{2} \widehat{R} L_{2} \widehat{R} & =\widehat{R} L_{2} \widehat{R} L_{2}, \\
J_{2} \widehat{R} L_{2} \widehat{R} & =\widehat{R} L_{2} \widehat{R} J_{2}, & J_{2} \widehat{R} J_{2} \widehat{R} & =-\widehat{R}^{-1} J_{2} \widehat{R} J_{2}
\end{aligned}
$$

Для того чтобы записать всю дифференциальную алгебру над $G L_{\mathrm{q}}(N)$, нам необходимо добавить образующие $T_{j}^{i}$ квантовой группы $\operatorname{Fun}\left(G L_{\mathrm{q}}(N)\right)$ с коммутационными соотношениями

$$
\begin{aligned}
\widehat{R} T_{1} T_{2} & =T_{1} T_{2} \widehat{R}, & \omega_{1} T_{2} & =T_{2} \widehat{R}^{-1} \omega_{2} \widehat{R}^{-1}, \\
J_{1} T_{2} & =T_{2} \widehat{R} J_{2} \widehat{R}, & L_{1} T_{2} & =T_{2} \widehat{R} L_{2} \widehat{R} .
\end{aligned}
$$

БРСТ-оператор $Q$ для дифференциальной алгебры (67)-(71) может быть построен с помошью формулы (55) и имеет следуюший вид [28]:

$$
\begin{aligned}
Q & =\operatorname{Tr}_{\mathrm{q}}\left(\omega \frac{L-\mathbf{1}}{\lambda}-\omega L(\omega J)+\lambda \omega L(\omega J)^{2}-\lambda^{2} \omega L(\omega J)^{3}+\cdots\right)= \\
& =\operatorname{Tr}_{\mathrm{q}}\left(\omega \frac{L-\mathbf{1}}{\lambda}-\omega L(\omega J)(\mathbf{1}+\lambda \omega J)^{-1}\right)=-\frac{1}{\lambda} \operatorname{Tr}_{\mathrm{q}}(\omega)+\frac{1}{\lambda} \operatorname{Tr}_{\mathrm{q}}(\Theta),
\end{aligned}
$$


где $\Theta=\omega L(\mathbf{1}+\lambda \omega J)^{-1}$, а $\operatorname{Tr}_{\mathrm{q}}(Y) \equiv \operatorname{Tr}\left(D^{-1} Y\right)$ обозначает квантовый след. Сумма в уравнении (72) конечна благодаря тому факту, что мономы по $\omega$ порядка $N^{2}+1$ равны нулю (т.к. внешняя алгебра форм (67) на квантовой группе $G L_{\mathrm{q}}(N)$ - это плоская деформация [6], [11] классической алгебры).

Можно непосредственно проверить, что БРСТ-оператор $Q$, заданный уравнением (73), удовлетворяет соотношениям

$$
\begin{aligned}
Q^{2}=0, & {[Q, L]=0, } \\
{[Q, T]=T \omega \equiv d T, } & {[Q, \omega]_{+}=-\omega^{2} \equiv d \omega } \\
{[Q, J]_{+}=} & \frac{1}{\lambda}(\mathbf{1}-L) .
\end{aligned}
$$

(Анти)коммутатор с БРСТ-оператором $Q$ (соотношения (75)) определяет действие дифференциала на алгебре $G L_{\mathrm{q}}(N)$ и ее внешнем расширении; это обеспечивает структуру комплекса де Рама $\Omega\left(G L_{\mathrm{q}}(N)\right)$ на подалгебре с образующими $T_{j}^{i}$ и $\omega_{j}^{i}$ (комплекс де Рама $\Omega\left(G L_{\mathrm{q}}(N)\right)$ впервые рассматривался Маниным, Мальтсиниотисом и Цыганом [6]).

Соотношение (76) - аналог тождества Картана. Для того чтобы получить соотношения (74)-(76), необходимо использовать свойство инвариантности квантового следа

$$
\operatorname{Tr}_{\mathrm{q}}(X) \mathbf{1}_{2}=\operatorname{Tr}_{\mathrm{q} 1}\left(\widehat{R}^{ \pm 1} X_{2} \widehat{R}^{\mp 1}\right)
$$

и соотношения

$$
\begin{gathered}
\widehat{R} \Theta_{2} \widehat{R}^{-1} \omega_{2}=-\omega_{2} \widehat{R}^{-1} \Theta_{2} \widehat{R}, \\
\widehat{R} \Theta_{2} \widehat{R}^{-1} \Theta_{2}=-\Theta_{2} \widehat{R}^{-1} \Theta_{2} \widehat{R}^{-1} \\
\widehat{R}^{-1} \Theta_{2} \widehat{R} L_{2}=L_{2} \widehat{R} \Theta_{2} \widehat{R}^{-1}, \quad \Theta_{1} T_{2}=T_{2} \widehat{R}^{-1} \Theta_{2} \widehat{R}, \\
J_{2} \widehat{R} \Theta_{2} \widehat{R}^{-1}+\widehat{R}^{-1} \Theta_{2} \widehat{R} J_{2}=-L_{2}(\mathbf{1}+\lambda \omega J)_{2}^{-1} \widehat{R}^{-1}(\mathbf{1}+\lambda \omega J)_{2},
\end{gathered}
$$

которые вытекают из (67)-(71).

ЗАмечАниЕ 1 . Оператор $Q$, заданный уравнением (73), имеет правильный классический предел при $q \rightarrow 1(\lambda \rightarrow 0, L \rightarrow \mathbf{1}+\lambda \widetilde{\chi}, \omega \rightarrow \widetilde{\omega}, J \rightarrow-\tilde{\gamma})$ :

$$
Q \rightarrow Q_{\mathrm{cl}}=\operatorname{Tr}\left(\widetilde{\omega} \widetilde{\chi}+\widetilde{\omega}^{2} \tilde{\gamma}\right)=\operatorname{Tr}(\widetilde{\omega} X-\widetilde{\omega} \tilde{\gamma} \widetilde{\omega}),
$$

где

$$
X \equiv \widetilde{\chi}+\widetilde{\omega} \tilde{\gamma}+\tilde{\gamma} \widetilde{\omega},
$$

и классическая алгебра определяется соотношениями

$$
\begin{gathered}
{\left[\widetilde{\omega}_{2}, \tilde{\gamma}_{1}\right]_{+}=P_{12}, \quad\left[\widetilde{\omega}_{2}, \widetilde{\omega}_{1}\right]_{+}=0=\left[\tilde{\gamma}_{2}, \tilde{\gamma}_{1}\right]_{+},} \\
{\left[X_{2}, X_{1}\right]=P_{12}\left(X_{2}-X_{1}\right),} \\
{\left[X_{2}, \widetilde{\omega}_{1}\right]=0=\left[X_{2}, \tilde{\gamma}_{1}\right] .}
\end{gathered}
$$


ЗАМЕчАНИЕ 2. Для дифференциального комплекса $\Omega\left(G L_{\mathrm{q}}(N)\right)$ можно определить группы когомологий де Рама $H^{p}\left(G L_{\mathrm{q}}(N)\right)$. Квантовые аналоги образующих кольца когомологий де Рама $H^{*}\left(G L_{\mathrm{q}}(N)\right)$ могут быть выбраны следуюшим образом:

$$
\Omega^{(n)}=\operatorname{Tr}_{\mathrm{q}}\left(\omega^{n}\right), \quad n=1,3,5, \ldots, 2 N-1
$$

Эти образуюшие удовлетворяют соотношениям [4], [12]

$$
d \Omega^{(n)}=-\operatorname{Tr}_{\mathrm{q}}\left(\omega^{n+1}\right)=0, \quad\left[\Omega^{(n)}, \Omega^{(k)}\right]_{+}=0 .
$$

5.2. Анти-БРСТ-оператор и квантовый лапласиан. По аналогии с тем, как мы построили явную формулу $(72),(73)$ для БРСТ-оператора $Q$, для алгебры $U_{\mathrm{q}}(g l(N))$ можно построить анти-БРСТ-оператор $Q^{*}$ :

$$
Q^{*}=\operatorname{Tr}_{\mathrm{q}}\left(J \frac{L^{-1}-\mathbf{1}}{\lambda}+J L^{-1} J \omega\right)=\frac{1}{\lambda}\left(\operatorname{Tr}_{\mathrm{q}}\left(\Theta^{*}\right)-\operatorname{Tr}_{\mathrm{q}}(J)\right)
$$

где $\Theta^{*}=J L^{-1}(\mathbf{1}+\lambda J \omega)$. Оператор $Q^{*}$ удовлетворяет соотношениям

$$
\begin{gathered}
\left(Q^{*}\right)^{2}=0, \quad\left[Q^{*}, L\right]=0, \\
{\left[Q^{*}, T\right]=q^{2 N} T J, \quad\left[Q^{*}, J\right]_{+}=-q^{2 N} J^{2},} \\
{\left[Q^{*}, \omega\right]=q^{2 N}\left(W \frac{1-L^{-1}}{\lambda} \bar{W}-\lambda \omega J^{2} \omega\right) .}
\end{gathered}
$$

Здесь фактор $q^{2 N}$ возникает в силу тождества (см. (66))

$$
\operatorname{Tr}_{\mathrm{q} 1}(R)=\operatorname{Tr}_{\mathrm{q} 1}\left(R^{-1}+\lambda \mathbf{1}\right)=q^{2 N} \mathbf{1}_{2}
$$

Для удобства мы введем новые матричные операторы [4]

$$
W=(\mathbf{1}+\lambda \omega J), \quad \bar{W}=(\mathbf{1}+\lambda J \omega) .
$$

Для вывода уравнений (87)-(89) мы использовали соотношения

$$
\begin{gathered}
\Theta_{1}^{*} T_{2}=T_{2} \widehat{R} \Theta_{2}^{*} \widehat{R}^{-1}, \quad \widehat{R}^{-1} \Theta_{2}^{*} \widehat{R} J_{2}=-J_{2} \widehat{R} \Theta_{2}^{*} \widehat{R}^{-1}, \\
\widehat{R} \Theta_{2}^{*} \widehat{R} \Theta_{2}^{*}=-\Theta_{2}^{*} \widehat{R} \Theta_{2}^{*} \widehat{R}^{-1}, \quad \widehat{R}^{-1} \Theta_{2}^{*} \widehat{R} L_{2}=L_{2} \widehat{R} \Theta_{2}^{*} \widehat{R}^{-1} \\
\widehat{R} \Theta_{2}^{*} \widehat{R}^{-1} \omega_{2}+\omega_{2} \widehat{R}^{-1} \Theta_{2}^{*} \widehat{R}=-\left(W L^{-1} \bar{W}\right)_{2} \widehat{R}
\end{gathered}
$$

Используя уравнения (88) и (89), можно определить дуальный дифференциал $d^{*}$ как (анти)коммутатор с анти-БРСТ-оператором $Q^{*}$. 
Теперь мы определим токовую матрицу $U_{j}^{i}$ :

$$
U \equiv W L^{-1} \bar{W}=-q^{-2 N}\left[\operatorname{Tr}_{\mathrm{q}}\left(\Theta^{*}\right), \omega\right]_{+},
$$

т.е. квантовый аналог матрицы $X$ (см. (81)). Матрица $U$ удовлетворяет уравнению отражения

$$
\widehat{R}^{-1} U_{2} \widehat{R}^{-1} U_{2}=U_{2} \widehat{R}^{-1} U_{2} \widehat{R}^{-1} .
$$

Для этой токовой матрищы справедливы коммутационные соотношения

$$
\widehat{R} U_{2} \widehat{R}^{-1} \omega_{2}=\omega_{2} \widehat{R}^{-1} U_{2} \widehat{R}, \quad \widehat{R}^{-1} U_{2} \widehat{R} J_{2}=J_{2} \widehat{R} U_{2} \widehat{R}^{-1}
$$

Это правильные квантовые аналоги соотношений коммутативности $(84)$ ( $U$ представляется в виде $U=\mathbf{1}+\lambda \mathbf{X}$, где $\mathbf{X} \rightarrow X$ при $q \rightarrow 1$ ), т.е.

$$
\mathbf{X}_{|1\rangle} \omega_{|2\rangle}=\sigma_{12} \omega_{|2\rangle} \mathbf{X}_{|1\rangle}, \quad \mathbf{X}_{|1\rangle} J_{|2\rangle}=\tilde{\sigma}_{12} J_{|2\rangle} \mathbf{X}_{|1\rangle},
$$

где $\sigma_{12}, \tilde{\sigma}_{12}$ - элементы алгебры кос, которые определяются соотношениями (92) и переходят в матрицу перестановки при $q=1$. Более того, преобразование левоинвариантного тока $U$ в правоинвариантный ток $\Xi$,

$$
\Xi=T W L^{-1} \bar{W} T^{-1}=T U T^{-1}
$$

ведет к условиям точной коммутативности $\left[\Xi_{2}, \omega_{1}\right]=0,\left[\Xi_{2}, J_{1}\right]=0$.

Квантовый оператор Лапласа определяется следующим образом (здесь достаточно воспользоваться соотношениями (74)-(76)):

$$
\Delta \equiv Q Q^{*}+Q^{*} Q=\lambda^{-2} \operatorname{Tr}_{\mathrm{q}}\left(L-2+L^{-1} \bar{W}-\lambda J L^{-1} \bar{W} \omega\right) .
$$

Лапласиан $\Delta$ инвариантен по отношению к БРСТ- и анти-БРСТ-операторам:

$$
[Q, \Delta]=0=\left[Q^{*}, \Delta\right]
$$

и обобщает квадратичный оператор Казимира для универсальной обертывающей алгебры $U_{\mathrm{q}}(g l(N))$.

Принимая во внимание тождество

$$
-\lambda \operatorname{Tr}_{\mathrm{q}}\left(J L^{-1} \bar{W} \omega\right)=\operatorname{Tr}_{\mathrm{q}}\left(\left(q^{2 N}-1\right) L^{-1} \bar{W}+\lambda q^{2 N} \omega J L^{-1} \bar{W}\right),
$$

можно получить замечательное выражение для квантового лапласиана через токовую матрицу $U$ :

$$
\Delta=\frac{1}{\lambda^{2}} \operatorname{Tr}_{\mathrm{q}}\left(L+q^{2 N} U-2\right)
$$


Теперь мы сформулируем теорему о разложении Ходжа. Рассмотрим пространство полиномов по переменным $\omega_{j}^{i}$ и $T_{j}^{i}$ с комплексными коэффициентами $\psi_{j_{1} \ldots j_{k}}^{i_{1} \ldots i_{k}}$,

$$
|\Psi\rangle=\Psi[T, \omega]=\sum_{k=0}^{K} \sum_{r=0}^{k} \operatorname{Tr}_{1 \ldots k}\left(T_{1} \ldots T_{r} \omega_{r+1} \ldots \omega_{k} \psi_{1 \ldots k}\right)
$$

(для некоторого $K$ ). Здесь случай $r=0$ соответствует произвольному полиному по $\omega$, который не зависит от образуюших $T$. Векторные поля $L_{j}^{i}-\delta_{j}^{i}$ и внутренние производные $J_{j}^{i}$ действуют слева на моном нулевого порядка $|0\rangle \equiv 1$ как операторы уничтожения:

$$
\left(L_{j}^{i}-\delta_{j}^{i}\right)|0\rangle=0, \quad J_{j}^{i}|0\rangle=0 .
$$

Это определяет левое действие БРСТ-, анти-БРСТ-операторов и оператора Лапласа на произвольные полиномы $|\Psi\rangle$. Теорема о разложении формулируется следуюшим образом.

ТЕОРемА. Произвольный полином $\Psi[T, \omega]$ может быть разложсен в сумму БРСТ-точного, коточного и гармонического полиномов:

$$
|\Psi\rangle=Q \cdot|\chi\rangle+Q^{*} \cdot|\Phi\rangle+|\Omega\rangle,
$$

где $\Delta|\Omega\rangle=0$

Доказательство этой теоремы проводится стандартным способом и аналогично доказательству теоремы о разложении для случая обычных алгебр Ли (см., например, $[17],[18])$.

Благодарности. Данная работа была частично поддержана грантом РФФИ № 9801-22033 и грантом CNRS, PICS-608. Работа А.П.И. была также частично поддержана грантом РФФИ № 00-01-00299.

\section{Список литературы}

[1] S. L. Woronowicz. Commun. Math. Phys. 1989. V. 122. P. 125.

[2] P. Aschieri, L. Castellani. Int. J. Mod. Phys. A. 1993. V. 8. P. 1667.

[3] A. Klimyk and K. Schmudgen. Quantum Groups and their Representations. Texts and Monographs in Physics. Part IV. Berlin: Springer, 1997.

[4] А. П. Исаев. ЭЧАЯ. 1997. Т. 28. № 3. С. 685.

[5] A. Connes. Noncommutative Geometry. New York: Academic Press, 1994.

[6] Ю. И. Манин. ТМФ. 1992. Т. 92. С. 425; G. Maltsiniotis. Commun. Math. Phys. 1993. V. 151. P. 275; C. R. Acad. Sci. 1990. V. 311. Sér. I. P. 831; B. Tsygan. Sel. Math. 1993. V. 12. P. 75 .

[7] B. Jurco. Lett. Math. Phys. 1991. V. 22. P. 177.

[8] Н. Ю. Решетихин, Л. А. Тахтаджкян, Л. Д. Фаддеев. Алгебра и анализ. 1989. Т. 1. № 1. C. 178 . 
[9] A. Sudbery. Phys. Lett. B. 1992. V. 284. P. 61; A. P. Isaev and P. N. Pyatov. Phys. Lett. A. 1993. V. 179. P. 81; hep-th/9211093.

[10] P. Schupp, P. Watts, and B. Zumino. Lett. Math. Phys. 1992. V. 25. P. 139.

[11] A. P. Isaev and P. N. Pyatov. J. Phys. A. 1995. V. 28. P. 2227; hep-th/9311112.

[12] L. D. Faddeev and P. N. Pyatov. The differential calculus on quantum linear groups. In: Contemporary Mathematical Physics. Eds. R. L. Dobrushin et al. Providence, RI: AMS, 1996. P. 35; hep-th/9402070; Л. Д. Фаддеев, П. Н. Пятов. Квантование дифференциального исчисления на линейных группах. В сб.: Проблемы современной теоретической физики, 96-212. Ред. А. П. Исаев. Дубна: ОИЯИ, 1996. С. 19.

[13] I. Ya. Aref'eva, G. E. Arutyunov, and P. B. Medvedev. J. Math. Phys. 1994. V. 35. № 12. P. 6658.

[14] G. E. Arutyunov, A. P. Isaev, and Z. Popowicz. J. Phys. A. 1995. V. 28. P. 4349; q-alg/9502002.

[15] U. Carow-Watamura, M. Schlieker, S. Watamura, and W. Weich. Commun. Math. Phys. 1991. V. 142. P. 605.

[16] M. Henneaux and C. Teitelboim. Quantization of Gauge Systems. Princeton: Univ. Press, 1992.

[17] J. W. van Holten. Nucl. Phys. B. 1990. V. 339. P. 158.

[18] C. Chryssomalakos, J. A. de Azcarraga, A. J. Macfarlane, and J. C. Perez Bueno. J. Math. Phys. 1999. V. 40. P. 6009.

[19] D. Bernard. Prog. Theor. Phys. Suppl. 1990. V. 102. P. 49; Phys. Lett. B. 1991. V. 260. P. 389.

[20] S. Watamura. Commun. Math. Phys. 1993. V. 158. P. 67.

[21] A. P. Isaev. J. Math. Phys. 1994. V. 35. P. 6784; hep-th/9402060; Lett. Math. Phys. 1995. V. 34. P. 333; hep-th/9403154.

[22] S. L. Woronowicz. Publ. RIMS Kyoto. 1987. V. 23. P. 117.

[23] T. Brzezinski. Lett. Math. Phys. 1993. V. 27. P. 287.

[24] Д. И. Гуревич. Алгебра и анализ. 1990. Т. 2. № 4. С. 119.

[25] O. V. Radko and A. A. Vladimirov. J. Math. Phys. 1997. V. 38. P. 5434.

[26] P. Schupp. Cartan calculus: differential geometry for quantum groups. hep-th/9408170.

[27] S. Baaj, G. Skandalis. Ann. Sci. Éc. Norm. Sup. 4 série. 1993. V. 26. P. 425; P. M. Kauaeв. Алгебра и анализ. 1996. Т. 8. № 4. С. 63; q-alg/9503005.

[28] C. Burdik, A. P. Isaev, and O. V. Ogievetsky. Standard complex for quantum Lie algebras. math.QA/0010060. 